\section{ORGANIC CHEMISTRY}

\section{Catalyst eases fuel production}

A catalyst could improve the manufacture of methanol, a promising fuel for renewable energy, from carbon dioxide. Current methods require high pressures or generate carbon monoxide, an undesirable by-product.

Jens Nørskov at Stanford University in California and his colleagues modelled the chemical reduction of $\mathrm{CO}_{2}$ to methanol at ambient pressure and identified nickel-galliumbased compounds as promising catalysts.

The researchers synthesized and tested a series of these catalysts, and found that $\mathrm{Ni}_{5} \mathrm{Ga}_{3}$ produced the same or larger amounts of methanol compared with conventional catalysts, while also generating less $\mathrm{CO}$, all at ambient pressure.

This catalyst could be used to make methanol as a fuel in, for instance, fuel cells, the authors say.

Nature Chem. http://doi.org/rss (2014)

\section{EVOLUTIONARY BIOLOGY}

\section{How the fish got its fins}

The adipose fin, which sits between the dorsal fin and the tail on many fishes, might have evolved separately in different fish lineages rather than once from a single ancestor. This suggests that the fin (pictured with arrow) has an adaptive purpose and can evolve into various forms, contrary to previous thinking.

Thomas Stewart at the University of Chicago, Illinois, and his colleagues reconstructed the evolutionary

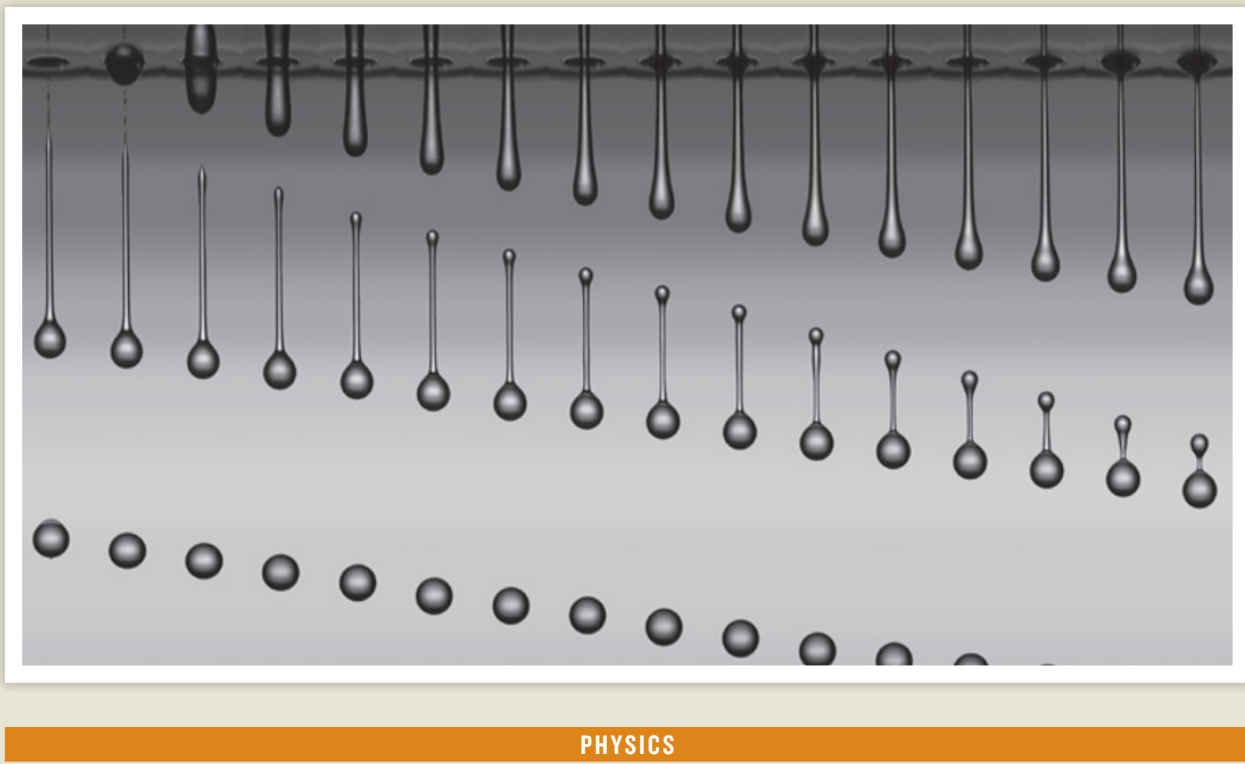

\title{
Fast imaging captures falling droplets
}

Researchers have obtained images of tiny oil droplets (pictured) forming in flight at high speeds. Such data on droplet formation could improve inkjet printing techniques.

Studying how droplets behave in inkjet printing is difficult because they move so rapidly. Detlef Lohse at the University of Twente in the Netherlands and his colleagues used 8-nanosecond-long flashes of a laser to light up picolitre-sized silicone oil droplets, and recorded these with a microscope and high-speed camera. By comparing two images of the same droplets taken 600 nanoseconds apart, the authors calculated the internal flow rate of the droplets as they formed, and found good agreement with mathematical simulations.

Phys. Rev. Applied 1, 014004 (2014) relationships of 232 fishes, looking at the presence and absence of adipose fins. They also studied the skeletons of 620 fish species from 55 families. The team concludes that these fins have a wide variety of skeletal structures and have repeatedly evolved some of the same features, such as fin rays - rods of bone or cartilage that

\section{support the in Egypt}

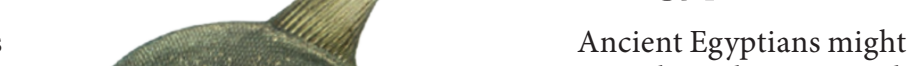
have domesticated wild cats nearly 2,000 years earlier than previously thought. Egyptian artwork from 4,000 years ago depicts domesticated cats alongside humans. But in 2008, Wim Van Neer at the Royal Belgian Institute of Natural Sciences in Brussels and his colleagues discovered six cat skeletons buried in a cemetery for elite Egyptians that dates to the fourth millennium вс. The teeth and bones resemble those of modern domestic felines. The cats - two pairs of kittens, and an older female and male - seem to have been born outside the breeding season of wild cats, suggesting that humans had a role in rearing them, the researchers say.

J. Arch. Sci. http://doi.org/rsg (2014) 